\title{
Evidence for Autosomal Recessive Inheritance of Infantile Dilated Cardiomyopathy: Studies from the Eastern Province of Saudi Arabia
}

\author{
MOHAMMED A. SELIEM, KHADER B. MANSARA, MAE PALILEO, XIAOBU YE, \\ ZHEN ZHANG, AND D. WOODROW BENSON
}

\begin{abstract}
Specialty Pediatrics Division, Saudi Aramco-Dhahran Health Center, Dhahran 31311, Saudi Arabia [M.A.S., K.B.M., M.P.]; and Division of Pediatric Cardiology [D.W.B.] and Department of Biometry and Epidemiology [X.Y., Z.Z.], Medical University of South Carolina, Charleston,
\end{abstract}

South Carolina 29425, U.S.A.

\begin{abstract}
Familial dilated cardiomyopathy is being increasingly recognized, but affected individuals $<10$ y are rarely identified. We describe the natural history of dilated cardiomyopathy and evaluate the mode of inheritance among infants of Arab descent from the Eastern Province of Saudi Arabia. We evaluated 55 consecutive cases of dilated cardiomyopathy in patients $<10 \mathrm{y}$ of age seen during a 5-y interval. Echocardiography was the primary diagnostic modality. The 55 cases represented $20 \%$ of the offspring of 41 families of Arab descent. In 19 families (46\%), parents were first cousins; there was no obvious consanguinity in 22 families (54\%). Age at presentation was $<30$ mo $(95 \%)$ (range, 1 to $100 \mathrm{mo})$; males $(38 \%)$ and females $(62 \%)$ were affected. Patients died ( 25 patients, $46 \%$ ), improved (15 patients, $27 \%$ ), or recovered (15 patients, 27\%). The left ventricular shortening fraction at diagnosis ranged from 5 to $28 \%$ and did not differ in those who died, improved, or recovered. Complex segregation analysis of the family data using the mixed model of inheritance showed that a model of recessive inheritance best fits the data. Recessively inherited dilated cardiomyopathy has been infrequently reported, perhaps because it may be difficult to
\end{abstract}

\section{ABSTRACT}

recognize in other patient groups in which consanguineous marriage is uncommon and the number of children per family is small. In the setting of consanguineous marriage, homozygosity mapping should lead to identification of the gene(s) causing dilated cardiomyopathy in the families we studied. (Pediatr Res 48: 770-775, 2000)

$\quad$ Abbreviations
LVSF\%, left ventricular shortening fraction
y, underlying liability scale
l, effect of single gene inheritance
c, effect of multifactorial
e, random environment effects
L, variance of dichotomous trait
C, variance of multifactorial component
E, variance of environmental component
LCAD, very-long-chain acyl-CoA dehydrogenase
Sd, standard deviation
NS, not significant
ECG, electrocardiogram

Despite improvements in therapy, dilated cardiomyopathy remains an important cause of morbidity and mortality in pediatric patients (1-10). Familial analyses are increasingly showing that dilated cardiomyopathy has a genetic or inherited basis, but, in the pediatric patient, the incidence and portion of cases with a familial cause have not been defined (11-27), and many cases are considered idiopathic (28). Autosomal dominant inheritance has been most frequently described in the familial forms of dilated cardiomyopathy, but, in such kin-

Received September 21, 1999; accepted June 30, 2000.

Correspondence: D. Woodrow Benson, M.D., Ph.D., Medical University of South Carolina, 114 Doughty St., Room \#223 ST Bldg., Charleston, SC 29425, U.S.A.

Reprint requests: Mohammed A. Seliem, M.D., Saudi Aramco-Dhahran Health Center, Dhahran 31311, Saudi Arabia.

Supported in part by NIH 1 P50 HL61006-01 (D.W.B.). dreds, affected individuals are rarely identified before the age of 10 y (12-19). The phenotype of X-linked cardiomyopathy associated with dystrophin mutations is usually identified in the adolescent or young adult male, whereas the X-linked cardiomyopathy associated with taffazin mutations (Barth's syndrome) typically onsets in infancy (20-23); taffazin mutations result in a highly lethal cardiomyopathy, but spontaneous recovery has been described (21-24). Dilated cardiomyopathy resulting from matrilinear (mitochondrial) inheritance is often noted because of associated neuromuscular manifestations (25, 26). Autosomal recessive inheritance of dilated cardiomyopathy has been infrequently described, perhaps because a familial association is more difficult to document (27), but fatty acid oxidation disorders resulting in dilated cardiomyopathy in young infants occur as recessive traits (1). 
The purpose of this study was to review all pediatric patients with idiopathic dilated cardiomyopathy who presented to Saudi Aramco-Dhahran Health Center in the previous $5 \mathrm{y}$. We identified 55 cases of dilated cardiomyopathy from 41 families. Complex segregation analysis of the family data using the mixed model of inheritance showed that a model of recessive inheritance best fits the data.

\section{METHODS}

Patient sample. We identified dilated cardiomyopathy by history, physical examination, and echocardiography. Echocardiographic indications of dilated cardiomyopathy were increased end-diastolic dimension and reduced LVSF\% (with or without right ventricle involvement) (2). Clinical manifestations consisted of signs and symptoms of acute congestive heart failure. During patient evaluation, we sought to identify known causes of acute heart failure, e.g. congenital heart disease, metabolic disease, or muscular dystrophy.

We identified 55 cases of dilated cardiomyopathy from 41 families. Forty-nine consecutive cases of dilated cardiomyopathy in patients age $14 \mathrm{y}$ or less (institutional definition of pediatrics) were seen at the Saudi Aramco-Dhahran Health Center during a 5-y interval; the remaining six cases were siblings of at least one of the 49 cases who died at other hospitals. Review of medical records of the deceased infants and careful questioning of family members unequivocally confirmed the cause of death as dilated cardiomyopathy in all six cases. Parents of patients were interviewed with regard to ethnic background, health history, and family history, which included parents, siblings, aunts, uncles, and first cousins of the probands.

Diagnostic evaluation. On presentation, each patient underwent complete physical examination, complete blood count and differential, urine analysis, serum glucose and electrolyte concentrations including calcium, phosphorus, and magnesium, chest x-ray examination, and 12-lead ECG. A comprehensive echocardiographic/Doppler examination was performed with either a Hewlett-Packard 1500 or 5500 echocardiographic system. Left ventricular dimensions and fractional shortening were obtained from M-mode tracing of the left ventricle just distal to the tips of the mitral valve leaflets in parasternal long axis view. All parameters were averaged from at least three cardiac cycles. All studies were conducted by the same technician (M.P.) and recorded on VHS format. Chloral hydrate sedation (up to $100 \mathrm{mg} / \mathrm{kg}$ ) was used as needed in children less than $3 \mathrm{y}$ of age.

A variety of additional laboratory tests were obtained in some patients, including serum carnitine (total and free) and selenium concentration, as well as liver function test, thyroid function tests, complete viral serology (Coxsackie A and B, influenza A and B, echoviruses, adenoviruses), and cytogenetic studies. Cardiac catheterization, endomyocardial biopsy, and autopsy were not performed on any patient.

Treatment and follow-up. Patient management varied over time and according to the presenting symptoms. All patients presented with congestive heart failure and respiratory distress and were routinely admitted to the Pediatric Intensive Care

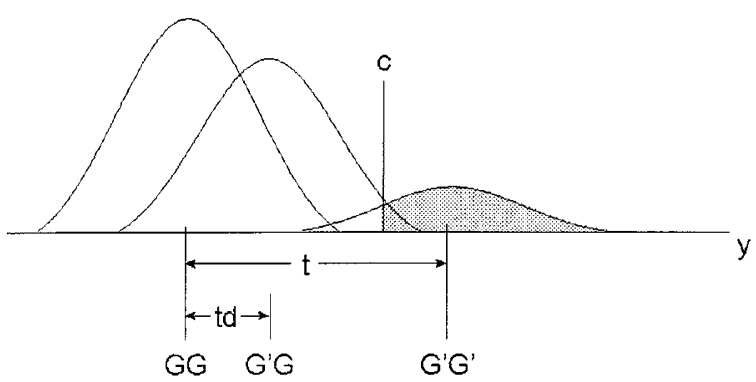

Figure 1. Mixed model of inheritance (autosomal). $G$ indicates normal allele; $G$ ', diseased allele; $t d$, displacement between the mean of the normal homozygote and the mean of the heterozygote; $C$, liability threshold for being affected.

Unit where they initially received inotropic drug support with dopamine, dobutamine, and/or amrinone, and sodium nitroprusside and furosemide. Supplemental $\mathrm{O}_{2}$ was used as needed. Intravenous gamma globulin was used in two patients and prednisone in two patients because a diagnosis of myocarditis could not be excluded.

Multiple echocardiograms were obtained serially on each patient. If patients became medically stable on the above regimen, oral anticongestive maintenance drug therapy was initiated. This included digoxin, furosemide, and/or captopril. Four patients received metoprolol, and two were treated with Coumadin after left ventricular thrombus was recognized. Patients with shortening fraction of $20 \%$ or less (laboratory normal is 28 to $40 \%)$ were given one baby aspirin $(100 \mathrm{mg})$ daily. Long-term follow-up at regular outpatient visits included complete physical examination, ECG, and echocardiograms as well as periodic serum electrolyte measurement. Holter monitoring was used when clinically indicated and was obtained in approximately half of the patients. Heart transplantation was not available to any patient.

Statistical analysis. $t$ test, 95\% confidence intervals, and $\chi^{2}$ analysis were used to compare the groups. The data were presented as mean $\pm \mathrm{SD}$. A $p$ value of $<0.05$ was considered statistically significant.

Complex segregation analysis was performed on the family data using the mixed model of inheritance (29). For a disease trait like cardiomyopathy, the model assumes that familial aggregation is due to an underlying liability scale (y) to which Mendelian inheritance of a single gene (l), multifactorial transmission (c), and random environment effects (e) contribute additively and independently, i.e. $\mathrm{y}=1+\mathrm{c}+$ e. For a dichotomous trait, 1 is assumed to have mean 0 and variance $\mathrm{L}$. The multifactorial component and the environmental component are assumed to be normally distributed with mean 0 and variance $\mathrm{C}$ and $\mathrm{E}$, respectively. Thus, the phenotype variance is the sum of the three components L, C, and E (Fig. 1). Ascertainment values were estimated from the distribution of the probands in our data. To determine which genetic model provided the best fit to the family data, analyses were conducted using the computer program POINTER (29), which compares the likelihood of a particular model (sporadic, multifactorial, recessive, or dominant) with the general model that incorporates single gene, multifactorial inheritance, and ran- 
dom environmental effects. Likelihood ratios $\left(\chi^{2}\right)$ are used as measures for goodness-of-fit. A smaller $p$ value indicates significant difference between a particular model and the general model with more parameters. If a particular model of inheritance was not significantly different from the general model, it was taken as the most parsimonious model. The most parsimonious model was chosen because it explains the data most efficiently with the least number of parameters.

\section{RESULTS}

Family history. Among the 41 families, the total number of offspring ranged from one to 14 (mean $\pm \mathrm{SD}=6.9 \pm 2.5$ ). One family had five affected offspring, three families each had four affected offspring, one family had two affected offspring (Fig. 2), and 36 families had a single affected offspring. All 82 parents were in excellent states of health without any evidence of heart disease, and dilated cardiomyopathy had not been recognized in any other family member. None of the 41 families was located through "pointers" (29).

Patient characteristics. There were 21 affected males and 34 affected females. Age at presentation was $<12$ mo (26 patients), $<24$ mo (49 patients), $<30$ mo (52 patients) (range, 1 to 100 mo; mean $\pm \mathrm{SD}=17.3 \pm 22$ ). At diagnosis, the
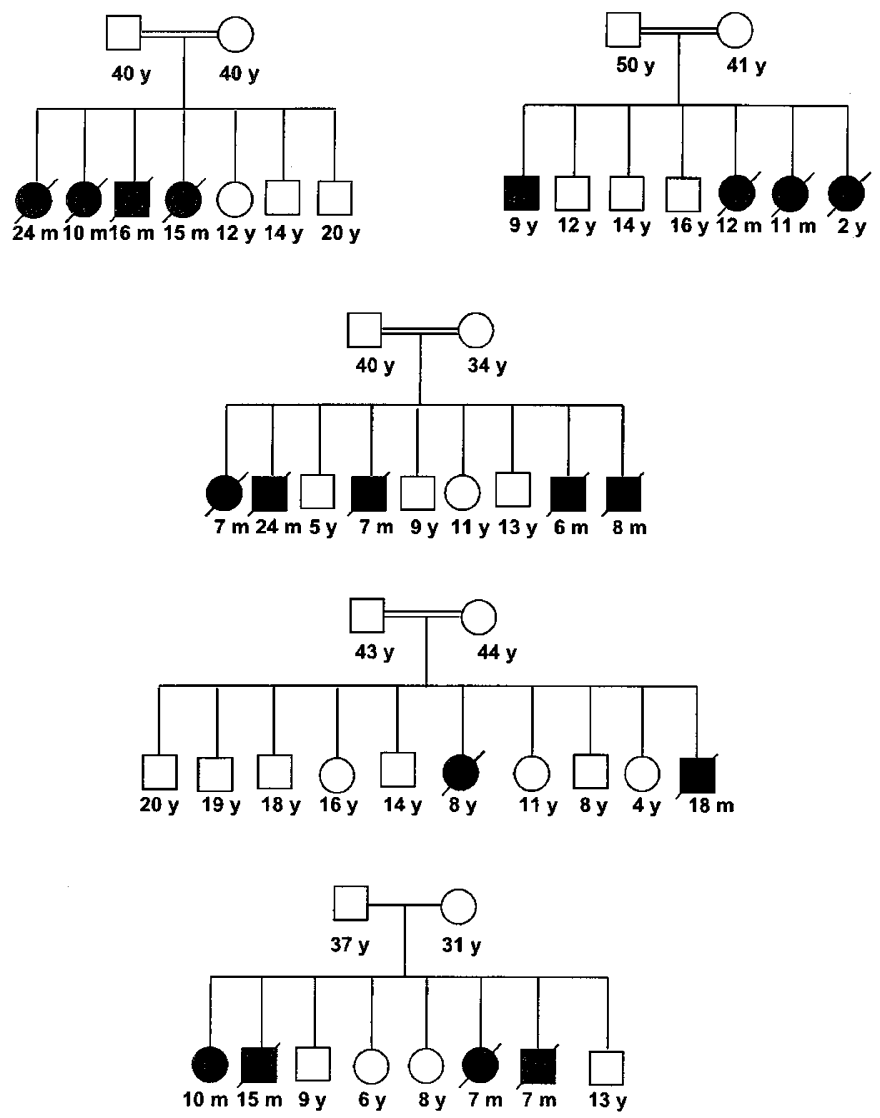

Figure 2. Pedigrees of five families with multiple affected infant siblings with dilated cardiomyopathy. Numbers are the ages at presentation for affected infants or age at initial assessment for unaffected family members ( $m$ indicates month; $y$, year). Blackened squares (males) and circles (females) indicate those affected with cardiomyopathy, and the diagonal line indicates those who died. Consanguinity is indicated by double bars between the parents.
LVSF\% ranged from 5 to $28 \%$. No patient had Kawasaki's disease, HIV, neutropenia, hypoglycemia, hypotonia, cataracts, or dysmorphic facial features. Fetal echocardiogram showing normal ventricular function was obtained during two pregnancies; both infants subsequently developed the disease at 3 and 12 mo of age.

Outcome. Based on clinical course and echocardiographic findings, patient outcome was determined to be death, recovery, or improvement. There was no difference in LVSF\% at the time of initial diagnosis among individuals who died, recovered, or improved. A fatal outcome occurred in 25 patients (46\%) from 13 families; this includes six cases that died at other hospitals. Thirteen infants died suddenly at home; those who died in hospital usually developed significant bradycardia progressing to fatal asystole. Mean age at death was $26.6 \pm$ $28.8 \mathrm{mo}$, range 7 to $103 \mathrm{mo}$, and $95 \%$ confidence interval 11.8-41.4. The mean interval of survival from the onset of the disease to death was $7.3 \pm 6.8$ mo (range, 1 to $24 \mathrm{mo}$ ), $95 \%$ confidence interval 4.0-10.6.

Cardiomyopathy was not fatal in 30 cases. In 15 patients (27\%), the LVSF\% returned to normal within 6 mo of diagnosis and remained so off all therapy. Within this group, the LVSF\% at diagnosis ranged from 5 to $21 \%$. In 15 patients (27\%), LVSF\% improved from the initial study while patients continued on maintenance drug therapy. The LVSF\% at diagnosis ranged from 7 to $22 \%$, and, at most recent follow-up, LVSF\% ranged from 10 to $26 \%$.

Other observations. No abnormality was noted in cytogenetic studies performed in 12 patients. Serum carnitine concentrations were normal in each patient. Viral titers were obtained in 16 patients, and there was evidence of viral infection in 11 patients: influenza A or B virus (seven patients), adenovirus (three patients), and Coxsackie B virus (one patient). No patient had abnormal body temperature elevation at the time of initial assessment or early in the disease course. X-ray study of the chest showed cardiomegaly in each patient. The ECG was normal or suggested left ventricular hypertrophy; isolated monomorphic premature ventricular contractions (five patients), left atrial enlargement (10 patients), and nonspecific ST-T wave changes (four patients) were present in some individuals. A variety of cardiac rhythm abnormalities were observed in patients during intensive care monitoring and included frequent multiform premature ventricular contractions, couplets and triplets, but there was no evidence of atrioventricular block or either atrial or ventricular tachycardia.

Demographics. All 82 parents and 282 offspring in the 41 families were born in the Eastern Province of Saudi Arabia. The original settlers of the Eastern Province were Bedouins (Arabs) (30). All family members were Arab, and their ancestors had always lived in this region. In 19 of 41 families (46\%), marriage was consanguineous (parents were first cousins), whereas, in 22 families (54\%), there was no obvious consanguinity. Three families had the same surname, but none knew of a relationship with any other family.

Comparison of cases based on presence or absence of consanguinity. Families were classified on the basis of the presence or absence of consanguinity. The average number of siblings per family was similar in families with consanguine- 
ous versus nonconsanguineous marriage, $7.2 \pm 2.7$ versus 6.6 $\pm 2.3, p=$ NS. In the 41 families, 55 of $282(20 \%)$ offspring developed dilated cardiomyopathy; 30 of 136 (22\%) offspring from consanguineous marriages were affected, whereas 25 of $146(17 \%)$ offspring from nonconsanguineous marriages were affected ( $p=\mathrm{NS}$ ). There were no statistically significant differences between cases from consanguineous versus nonconsanguineous marriage in age at diagnosis or gender distribution. Fatal outcome (death) occurred in 18 infants from eight consanguineous marriages and seven infants from five nonconsanguineous marriages $(p=0.036)$. Based on the outcome [dead $(n=25)$ versus recovered/improved $(n=30)$ ], there was no difference in age at presentation or gender. Elevated viral titers were observed in six individuals from consanguineous marriages (three deaths) and five individuals from nonconsanguineous marriages (three deaths).

Segregation analysis. Among offspring from consanguineous marriages, the proportion of affected siblings was close to the $25 \%$ predicted by classic autosomal recessive inheritance. On the basis of the similarity of the dilated cardiomyopathy phenotype, the ethnic background of families, and the proportion of affected offspring among consanguineous versus nonconsanguineous marriages, we hypothesized that most cases of dilated cardiomyopathy might be the result of autosomal recessive inheritance. We used complex segregation analysis to test this possibility.

The families were divided into two groups according to the consanguinity of marriage. Tables 1 and 2 present the estimated parameters of fitted models from segregation analysis for dilated cardiomyopathy of children from consanguineous and nonconsanguineous marriages, respectively. Due to the fact that all the parents in the data set are apparently unaffected with dilated cardiomyopathy and none of the families was identified through pointers, parameters associated with the multifactorial transmissible components were not included in the model fitting process. The most general model, therefore, is the one in which parameters $\mathrm{d}, \mathrm{t}$, and $\mathrm{q}$ are all estimated through iterations. The parameter $\mathrm{q}$ in the mixed model is the frequency of the diseased allele.

The results show, for both consanguineous and nonconsanguineous marriage groups, that the estimated parameters of the recessive models are almost identical to that of the more general model in which the parameter $d$ was not fixed in iteration $(p>0.99)$. Therefore, the recessive model was selected as the most appropriate model because it has a smaller number of parameters. For the nonconsanguineous group, the additive model is rejected due to its fairly significant difference from the general model ( $p<0.1)$. The fitting of a dominant model failed with data from the nonconsanguineous group. The POINTER program reported the error of "peculiar boundary value reached" during iteration. For the consanguineous group, the dominant model is easily rejected due to its significant difference from the general model $(p<0.01)$. In this analysis, the interest is to determine models with fewer parameters that are most similar to the general models with more parameters. The additive model, even though it has $p=0.36$, is still not as close to the general model as the recessive one.

\section{DISCUSSION}

Findings in this report provide compelling evidence that in the families we studied, dilated cardiomyopathy that affects males and females at a relatively young age $(95 \%<30$ mo of age) is inherited in an autosomal recessive fashion. Several observations support this conclusion. First, parents are descended from a common ethnic background, and, in approximately one half of the families, offspring result from consanguineous marriages. Additionally, the proportion of affected offspring is close to the $25 \%$ predicted by autosomal recessive inheritance. Although results of the segregation analysis confirm our hypothesis that in these families most cases of dilated cardiomyopathy result from recessive inheritance, the limitation of the data set does not allow us to fully assess the effect of multifactorial transmission. The results of this study indicate the need for additional data, probably through ascertaining additional relatives of families in this study and their offspring.

The possibility that some cases of dilated cardiomyopathy we evaluated are due to other genetic causes including mitochondrial disease or environmental causes such as myocarditis cannot be excluded. For example, on the basis of presentation at a much older age (90-100 mo), three individuals may be suspected of having cardiomyopathy due to another cause. Two individuals with older age of cardiomyopathy onset were the only affected offspring from a nonconsanguineous marriage and may represent sporadic cases. However, a third individual, diagnosed at 90 mo of age, was the offspring of a consanguineous marriage and had an affected sibling who presented at 11 mo of age; findings in this latter case are compatible with recessive inheritance of dilated cardiomyopathy with delayed age of onset. It was not possible to find a grouping of patients consistent with different etiologies based on age at presentation or other aspects of clinical status.

It has long been known that some pediatric patients with dilated cardiomyopathy live a long time in a relatively stable

Table 1. Segregation analysis of DCM of children from consanguineous marriages

\begin{tabular}{lcrrrr}
\hline Model & $\mathrm{D}$ & $\mathrm{t}$ & $\mathrm{Q}$ & $\chi^{2}(d f=1)$ & \multicolumn{1}{c}{$p$} \\
\hline Recessive & $0.00^{*}$ & 8.3364091 & 0.0038053 & 0.000 & 0.827 \\
Additive & $0.50^{*}$ & 11.7054983 & 0.0037895 & 6.99 & 0.988 \\
Dominant & $1.00^{*}$ & 4.0579436 & 0.0000157 & 0.0038011 & \\
General & $0.00 \dagger$ & 8.3694817 & & \\
\hline
\end{tabular}

* Parameter was set and not iterated.

$\dagger$ Parameters went to bound.

$\mathrm{d}=$ dominance; $\mathrm{q}=$ gene frequency of the allele leading to affection; $\mathrm{t}=$ difference between the means of the liability distribution of the two homozygous classes, so that "dt" is the displacement between the mean of the normal homozygote and the mean of the heterozygote. 
Table 2. Segregation analysis of DCM of children from nonconsanguineous marriages

\begin{tabular}{lccccc}
\hline Model & $\mathrm{D}$ & $\mathrm{t}$ & $\mathrm{Q}$ & $\chi^{2}(d f=1)$ & $p$ \\
\hline Recessive & $0.00^{*}$ & 7.9043019 & 0.0017812 & 0.000 \\
Additive & $0.50^{*}$ & 10.9642835 & 0.0022603 & 2.811 \\
Dominant & $1.00^{*}$ & & & fitting failed \\
General & $0.00 \dagger$ & 7.9067532 & 0.0017724 & $<0.10$ \\
\end{tabular}

* Parameter was set and not iterated.

$\uparrow$ Parameters went to bound.

clinical state and may even appear to recover, whereas others have progressive myocardial dysfunction. Age at diagnosis, histologic findings, and echocardiographic features have limited predictive value (3-10). Even a genetic diagnosis may have limited predictive value of outcome. For example, patients with X-linked dilated cardiomyopathy due to dystrophin mutations have a progressive course leading to medically refractory heart failure, whereas some patients with X-linked cardiomyopathy due to mutations in tafazzin may have severe or lethal cardiac disease during infancy and early childhood but show relative improvement in later childhood. Recent reports of individuals with mutations in VLCAD, a catalyst in the first step of the $\beta$-oxidation spiral of fatty acid metabolism, have provided additional observations that young patients with genetically acquired dilated cardiomyopathy may die, improve, or recover (31). Nearly $40 \%$ of individuals with cardiomyopathy due to homozygous or compound heterozygous mutations in VLCAD recover with favorable long-term outlook (31).

Identification of individuals at highest risk of developing refractory heart failure may be of practical clinical use in that the need for cardiac transplantation may be identified early in the disease course. Among the families we studied, findings are compatible with autosomal recessive inheritance, but affected children were more likely to die if they were offspring of a consanguineous marriage. In this context, the difference in disease severity may result from whether an individual is a homozygote (consanguineous marriage) versus a compound heterozygote (nonconsanguineous marriage).

Infants who develop dilated cardiomyopathy are apparently born with completely normal hearts. At a relatively young age, they develop sudden onset of severe dilated cardiomyopathy. Approximately $50 \%$ of these patients die, usually early in the disease course. The remaining patients split more or less equally between those who seem to recover completely and those with residual left ventricular dysfunction requiring longterm medical treatment. Our current practice is to support these patients with maximal conventional medical therapy and follow them closely with frequent echocardiograms. Those who continue to have markedly depressed LVSF\% beyond 6 mo (usually $<15 \%$ ) continue to have chronic left ventricular dysfunction or die. These patients may benefit most from early referral for heart transplantation.

This report is a retrospective study reflecting the clinical experience of a single tertiary referral center with all the inherent deficiencies of such a study. We were not able to echo parents or apparently unaffected offspring or other relatives including cousins, aunts, or uncles. The follow-up on individuals who recovered or stabilized is relatively short; and we do not know if these individuals who were critically ill but recovered will have similar episodes later in life. We were not able to obtain tissue diagnosis on any of our patients (no endomyocardial biopsy or autopsy). However, these studies provide an opportunity to learn more about dilated cardiomyopathy due to autosomal recessive inheritance that may be difficult to recognize in other patient groups in which consanguineous marriage is uncommon and the number of children per family is small.

A number of important differences in phenotype of the affected individuals that we evaluated make it unlikely that they have a previously described defect in the $\beta$-oxidation pathway of long-chain fatty acids $(1,31)$. However, similarities, including recessive inheritance and occurrence of dilated cardiomyopathy in infants and young children who exhibit a significant percentage of spontaneous recovery, suggest that deficiency of a component of another metabolic pathway may be a candidate gene for the cause of cardiomyopathy in the families we studied. In children of consanguineous marriage, it is reasonable to assume that the disease gene is contained in a genetic locus that is homozygous by descent. In this situation, homozygosity mapping has been shown to be a practical way to map a recessive disease gene by studying a relatively small number of unrelated affected offspring of consanguineous marriage (32).

Acknowledgments. The authors wish to acknowledge the use of Saudi Aramco Medical Services Organization facilities for the data and study, which resulted in this paper. Drs. Seliem and Mansara and Mae Palileo were employed by Saudi Aramco during the time the study was conducted and the paper written.

Segregation analysis was accomplished using the computer program POINTER, software licensed by the Biomolecular Computing Resource, an element of MUSC's University Research Resource Facility.

\section{REFERENCES}

1. Kelly DP, Strauss AW 1994 Inherited cardiomyopathies. N Engl J Med 330:913-919

2. Schwartz ML, Cox GF, Lin AE, Korson MS, Perez-Atayde A, Lacro RV, Lipshultz SE 1996 Clinical approach to genetic cardiomyopathy in children. Circulation 94:2021-2038

3. Akagi T, Benson LN, Lightfoot NE, Chin K, Wilson G, Freedom RM 1991 Natural history of dilated cardiomyopathy in children. Am Heart J 121:1502-1506

4. Friedman RA, Moak TP, Garson Jr A 1991 Clinical course of idiopathic dilated cardiomyopathy in children. J Am Coll Cardiol 18:152-156

5. Gersony WM 1991 The child with dilated cardiomyopathy: prognostic consideration and management decisions. J Am Coll Cardiol 18:157-158

6. Lewis AB, Chabot M 1991 Outcome of infants and children with dilated cardiomyopathy. Am J Cardiol 68:365-369

7. Wiles HB, McArthur PD, Taylor AB, Gillette PC, Fyfe DA, Mathews JP, Shelton LW 1991 Prognostic features of children with idiopathic dilated cardiomyopathy. Am J Cardiol 68:1372-1376 
8. Ciszewski A, Biliniska ZT, Lubiszewska B, Ksiezycka E, Poplawska W, Michalak E, Walczak E, Walczak F, Ruzyllo W 1994 Dilated cardiomyopathy in children: clinical course and prognosis. Pediatr Cardiol 15:121-126

9. Griffin ML, Hernandez A, Martin TC, Goldring D, Bolman RM, Spray TL, Strauss AW 1988 Dilated cardiomyopathy in infants and children. J Am Coll Cardiol 11:139-144

10. Matitiau A, Perez-Atayde A, Sanders SP, Sluysmans T, Parness IA, Spevak PJ, Colan SD 1994 Infantile dilated cardiomyopathy: relation of outcome to left ventricula mechanics, hemodynamics, and histology at the time of presentation. Circulation 90:1310-1318

11. Michels VV, Moll PP, Miller FA, Tajik AJ, Chu JS, Driscoll DJ, Burnett JC, Rodeheffer RJ, Cheseboro JH, Tazelaar HD 1992 The frequency of familial dilated cardiomyopathy in a series of patients with idiopathic dilated cardiomyopathy. N Engl J Med 326:77-82

12. Kass S, MacRae C, Graber HL, Sparks EA, McNamara D, Boudoulas H, Basson CT, Baker PB, Cody RT, Fishman MC, Cox N, Kong A, Wooley CF, Seidman JG, Seidman CE 1994 A gene defect that causes conduction system disease and dilated cardiomyopathy maps to chromosome 1p1-1q1. Nat Genet 7:546-551

13. Olson TM, Keating MT 1996 Mapping a cardiomyopathy locus to chromosome 3p22-p25. J Clin Invest 97:528-532

14. Messina DN, Speer MC, Pericak-Vance MA, McNally EM 1997 Linkage of familia dilated cardiomyopathy with conduction defect and muscular dystrophy to chromosome 6q23. Am J Hum Genet 61:909-917

15. Durand JB, Bachinski LL, Bieling LC, Czernuszewicz GZ, Abchee AB, Yu QT, Tapscott T, Hill R, Ifegwu J, Marian AJ, Brugada R, Gregortich JM, Anderson J, Quinones M, Towbin JA, Roberts R 1995 Localization of a gene responsible for familial dilated cardiomyopathy to chromosome 1q32. Circulation 92:3387-3389

16. Krajinovic M, Pinamonti B, Sinagra G, Vatta M, Severini GM, Milasin J, Falaschi A Camerini F, Giacca M, Mestroni L 1995 Linkage of familial dilated cardiomyopathy to chromosome 9. Am J Hum Genet 57:846-852

17. Bowles KR, Gajarski R, Porter P, Goytia V, Bachinski L, Roberts R, Pignatelli R, Towbin JA 1996 Gene mapping of familial autosomal dominant dilated cardiomyopathy to chromosome 10q21-23. J Clin Invest 98:1355-1360

18. Siu BL, Niimura H, Osborne JA, Fatkin D, MacRae C, Solomon S, Benson DW, Seidman JG, Seidman CE 1999 A familial dilated cardiomyopathy locus that maps to chromosome 2q31. Circulation 99:1022-1026

19. Olson TM, Michels VV, Thibodeau SN, Tai YS, Keating MT 1998 Actin mutations in dilated cardiomyopathy, a heritable form of heart failure. Science 280:750-752
20. Towbin JA, Hejtmancik JF, Brink P, Gelb B, Zhu XM, Chamberlin JS, McCabe ER, Swift M 1993 X-linked dilated cardiomyopathy. Molecular genetic evidence of linkage to the Duchenne muscular dystrophy (dystrophin) gene at the Xp21 locus. Circulation 87:1854-1865

21. Barth PG, Scholte HR, Berden JA, Van der Klei-Van Moorsel JM, Luyt-Houwen IE, Van't Veer-Korthof ET, Van der Harten JJ, Sobotka-Plojhar MA 1983 An X-linked mitochondrial disease affecting cardiac muscle, skeletal muscle, and neutrophil leucocytes. J Neurol Sci 62:327-355

22. Bolhuis PA, Hensels GW, Hulsebos TJ, Baas F, Barth PG 1991 Mapping of the locus for X-linked cardioskeletal myopathy with neutropenia and abnormal mitochondria (Barth syndrome) to Xq28. Am J Hum Genet 48:481-485

23. Bione S, D'Adamo P, Maestrini E, Gedeon AK, Bolhuis PA, Toniolo D 1996 A nove X-linked gene, G4.5, is responsible for Barth syndrome. Nature Genet 2:385-389

24. D'Adamo P, Fassone L, Gedeon A, Janssen EA, Bione S, Bolhuis PA, Barth PG, Wilson M, Haan E, Orstavik KH, Patton MA, Green AJ, Zammarchi E, Donati MA, Toniolo D 1997 The X-linked gene G4.5 is responsible for different infantile dilated cardiomyopathies. Am J Hum Genet 61:862-867

25. Zeviani M, Gellera C, Antozzi C, Rimoldi M, Morandi L, Villani F, Tiranti V, DiDonato S 1991 Maternally inherited myopathy and cardiomyopathy: association with mutation in mitochondrial DNA tRNA (Leu) (UUR). Lancet 338:143-147

26. Tanaka M, Ino H, Ohno K, Hattori K, Sato W, Ozawa T, Tanaka T, Itoyama S 1990 Mitochondrial mutation in fatal infantile cardiomyopathy. Lancet 336:1452

27. Goldblatt J, Melmed J, Rose AG 1987 Autosomal recessive inheritance of idiopathic dilated cardiomyopathy in a Madeira Portuguese kindred. Clin Genet 31:249-254

28. Online Mendelian Inheritance in Man, OMIM (TM). Johns Hopkins University, Baltimore, MD. World Wide Web URL:http://www3.ncbi.nlm.nih.gov/omim/

29. Lalouel JM, Morton NE 1981 Complex segregation analysis with pointers. Hum Hered 31:312-321

30. Farag TI, Teebi AS 1997 Genetic disorders among the Bedouins. In: Farag TI, Teebi A (eds) Genetic Disorders among Arab Populations. Oxford University Press, New York, pp 375-410

31. Mathur A, Sims HF, Gopalakrishnan D, Gibson B, Rinaldo P, Vockley J, Hug G, Strauss AW 1999 Molecular heterogeneity in very-long-chain acyl-CoA dehydrogenase deficiency causing pediatric cardiomyopathy and sudden death. Circulation 99:1337-1343

32. Lander ES, Botstein D 1987 Homozygosity mapping: a way to map human recessive traits with the DNA of inbred children. Science 236:1567-1570 